\title{
EL TUTOR PEDAGÓGICO: ESLABÓN DETERMINANTE DEL PROCESO DE ENSEÑANZA APRENDIZAJE
}

MS.c Laura Jazmín Marín Granados Docente

Universidad de Guayaquil

lauraj.marin@outlook.com
MS.c. Yaneiris Castro Durand Docente Instituto Tecnológico de FormaciónProfesional yaneiris.castro@formacion.edu.ec
MS.c Alicia Valentina Álvarez Pérez Docente Instituto Tecnológico de Formación Profesional alicia.alvarez@formacion.edu.ec

Palabras claves: proceso de enseñanza-aprendizaje, tutor pedagógico, proceso de nivelación

Recibido: 5 de abril de 2016

Keywords: teaching-learning process, pedagogical tutor, leveling process

Aceptado: 21 de junio de 2016

\section{RESUMEN}

Dentro de la política del Ministerio de Educación y en aras de perfeccionar el proceso de enseñanza aprendizaje para los estudiantes que salen del nivel bachillerato y se disponen a ingresar a la universidad, luego de cumplir con los requisitos establecidos y realizar las pruebas pertinentes, surge el proyecto de Nivelación de Carrera, sustentado y basado bajo las normas y reglamentos del SNNA (Sistema Nacional de Nivelación y Admisión). Este proyecto tiene como particularidad que dentro de su personal profesional docente surge la figura del Tutor Pedagógico, el mismo que centra su accionar en dar un acompañamiento y seguimiento al estudiante durante este período, además de brindarle las herramientas investigativas para que estos al final del proceso puedan elaborar un Proyecto Integrador de Saberes como instrumento de culminación de estudio. El presente artículo proponer un conjunto de acciones pedagógicas que permitan fortalecer y orientar el trabajo del tutor pedagógico en el proceso de enseñanza aprendizaje en la Nivelación de Carrera.

\section{ABSTRACT}

As part pof the policies of the ministry of Education and with the objective of improving the teaching -learning process for those students graduating from school and prepare to enter college , after fulfiling the established requirements, the leveling career proyect was developed, based on the rules of SNNA (National System of leveling and Admission ) The proyect has a peculiarity of including within its profesional staff the pedagogical tutor, encharged of accompanying and following the student during this period. The tutor also offers the research tools for them to prepare a proyect at the end of this leveling period, the present articles proposes some pedagogical actions that alow strengtehning and orienting the tutor's work in the teaching -learning process in the leveling career proyect. 


\section{INTRODUCCIÓN}

Los momentos actuales que vive la educación demuestran la necesidad de implementar cambios que deben ser insertados en la práctica pedagógica, pues se exigen transformaciones en la concepción tradicional de la escuela de hoy, esto significa, que el nuevo ambiente no se queda en lo fenoménico, sino que expresa en lo esencial un proceso de enseñanza-aprendizaje que requiere de estrategias o acciones educativas, estilos de dirección, métodos acordes con las exigencias de las propias transformaciones, lo que requiere, además, de un educador profesional más preparado, capacitado para asumir las nuevas exigencias de su actividad formativa.

Pedagogos, investigadores y otros estudiosos de esta ciencia centran sus esfuerzos en la búsqueda de nuevas vías y acciones que mejoren la calidad de la enseñanza y que se implementen nuevos y dinámicos métodos de enseñanza, con el fin de alcanzar la calidad de este proceso, ya que es la misión y objetivo que persiguen de todas las instituciones y unidades educativas, vinculándose este con la eficiencia con que se enseña, se diagnostica, se caracteriza y se evalúa a los estudiantes.

El rol del docente suele ser redimensionado siempre y cuando se requiera, pues está centrado en orientar la formación y la enseñanza de sus educandos, darles seguimiento en este proceso, estableciendo mecanismos de control que le permitan conocer cómo se produce éste y desarrollar la evaluación con sistematicidad para continuar la orientación o reorientación, de aquellos saberes en los que, el compromiso afectivo matiza el reto del proceso de calidad de la educación, con soporte en la ciencia pedagógica y constituye un desafío para el Sistema Nacional de Educación.

Para lograr calidad en el proceso de enseñanza aprendizaje se requiere la implementación de guías de control y supervisión al trabajo que realizan los docentes, pues no todo debe quedar a la espontaneidad y más cuando de ellos depende la formación de las nuevas generaciones que tienen su encargo social establecido y son los medidores de la calidad del nuevo proceso educacional que se implementa en la nueva sociedad.

El proceso de enseñanza actual y las nuevas investigaciones del tema han demostrado que al abordar el fracaso o éxito en el sistema educacional debe de tenerse en cuenta dentro de sus parámetros la calidad con que se desempeñan los docentes. Además de otros factores como el perfeccionamiento de los programas y planes de estudios, pero solo que no se cuente con los docentes preparados, dotados de la didáctica y la pedagogía, eficaces y con destrezas no se podrá contar entonces con el perfeccionamiento de la educación, en aras de alcanzar su calidad.

Dentro de la política del Ministerio de Educación y en aras de perfeccionar el proceso de enseñanza aprendizaje para los estudiantes que salen del nivel bachillerato y se disponen a ingresar a la universidad, luego de cumplir con los requisitos establecidos y realizar las pruebas pertinentes, surge el proyecto de Nivelación de Carrera, sustentado y basado bajo las normas y reglamentos del SNNA (Sistema Nacional de Nivelación y Admisión).

Este proyecto tiene como particularidad que dentro de su personal profesional docente surge la figura del Tutor Pedagógico, el mismo que centra su accionar en dar un acompañamiento y seguimiento al estudiante durante este período, además de brindarle las herramientas investigativas para que estos al final del proceso puedan elaborar un Proyecto 
Integrador de Saberes como instrumento de culminación de estudio. El presente artículo proponer un conjunto de acciones pedagógicas que permitan fortalecer y orientar el trabajo del tutor pedagógico en el proceso de enseñanza aprendizaje en la Nivelación de Carrera.

\section{DESARROLLO}

El período de Nivelación de Carrera surge como un proyecto en el año 2012 que se desarrollaría Provincia del Guayas, Cantón Guayaquil y con un área de influencia en las distintas facultades que posee la Universidad de Guayaquil, dígase la ciudadela universitaria, el complejo de Párvulos de la Facultad de Filosofía, las Facultades de Industrial, FACSO, Ciencias Naturales, Ingeniería en Sistemas y demás recintos donde se desarrollan las actividades académicas de dicha institución.

El Curso de Nivelación es concebido como uno de los componentes que posee el Sistema Nacional de Nivelación y Admisión que desde su inicio tuvo una proyección teórico-metodológica que estaba orientada a establecer las estructuras, estrategias curriculares y organización del aprendizaje básico, con los siguientes objetivos:

\section{OBJETIVO GENERAL}

Optimizar las capacidades de aprendizaje de los aspirantes al ingreso a las Instituciones de Educación Superior desarrollando habilidades, destrezas, competencias y desempeños necesarios para que asuman el conocimiento disciplinar, tecnológico, profesional y humanístico de forma responsable y exitosa, desde el ejercicio del derecho a una educación de calidad y en igualdad de oportunidades educativas (Santos, 2012).

Igualmente se establecieron los Objetivos Específicos como puntos de partida para el trabajo a realizar (Santos, 2012):

- Desarrollar en los estudiantes habilidades de comunicación oral y escrita, en el marco del desarrollo de sus capacidades para el manejo de los diversos lenguajes de la ciencia, la profesión y las humanidades.

- Propiciar contextos educativos que fortalezcan las habilidades básicas del pen samiento y la lógica de razonamiento para la formulación estratégica de problemas y desarrollo del pensamiento abstracto.

- Fortalecer los sistemas conceptuales básicos de los campos disciplinares y hu manísticos que sustentan la profesión, permitiendo el desarrollo del pensamiento científico y crítico.

- Consolidar las metodologías de aprendizaje, competencias y habilidades nece sarias para la producción e interpretación de problemas vinculados al campo de la profesión.

- Promover la construcción del proyecto de vida personal, profesional y ciudadano de los estudiantes, con miras a fortalecer sus procesos de identidad, auto-organi zación y reflexividad. 
Si bien los objetivos expresan claramente la finalidad de este curso nivelatorio, es importante resaltar que la nivelación de carrera no busca fomentar y potenciar capacidades de su carrera a fin en la universidad, sino que se encamina a fortalecer las capacidades que debieron ser adquiridas en su etapa de bachillerato, es decir, al recibir al estudiantado de diversas instituciones, se trabajaría en equilibrar y nivelar los conocimientos que traerían y que realmente debían poseer.

Es por ello que en el año 2012 se procede a Institucionalizar el Departamento de Nivelación y Admisión en la Universidad de Guayaquil de modo que permita garantizar el antecedente formativo de calidad y articule con la oferta académica, el modelo educativo, las políticas institucionales, las trayectorias contextuales y las demandas formativas de los sujetos que cursarán la formación profesional en dicha IES.

Dentro de este proyecto establecido y que hasta la fecha muestra resultados positivos se encuentra un componente importante y es el objeto de estudio de la presente investigación y es Asesoría y Tutoría Pedagógica Estudiantil durante este período de seis meses.

Según la autora Josefina Santos, la Asesoría y Tutoría pedagógica es un componente del proceso de enseñanza aprendizaje durante este período que irá orientado al acompañamiento estudiantil de los jóvenes que ingresan a la universidad sin mayores fortalezas operacionales, cognitivas y actitudinales, que le permitan enfrentar los desafíos académicos e iniciar un proceso de inserción en el campo universitario (Santos, 2012).

Cada carrera deberá tener un asesor pedagógico estudiantil, que será un profesor tiempo completo el mismo que cumpla con el perfil por competencias que se ha establecido y que es conocido como la figura de TUTOR PEDAGÓGICO.

Según el instructivo que rectorea el trabajo del Departamento de Nivelación de Carrera y de manera específica el trabajo del Tutor Pedagógico, establece las siguientes funciones a cumplir por este profesional (Santos, 2012):

- Recabar información sobre las dificultades de aprendizaje y el rendimiento académico.

- Generar espacios de diálogo y trabajo en equipo para la revisión de los procesos de aprendizaje en las asignaturas críticas.

- Programar sesiones individuales o grupales para fortalecer las competencias del estudiante en el desarrollo del trabajo intelectual y académico.

- Planificar y definir objetivos con los estudiantes que enfrenten problemas de rendimiento académico promoviendo su autonomía en el diseño de su propio proyecto de estudio.

- Organizar una red de estudiantes de los últimos semestres que apoyen a los estudiantes de primer y segundo ciclo que están con dificultades académicas (estas horas de trabajo serán asumidas como de práctica de servicio comunitario).

- Ejecutar acciones de seguimiento continuo de orientación y apoyo en los procesos académicos. 


\section{- Promover la motivación continua de los estudiantes}

Partiendo de lo establecido por la Dirección técnica de Nivelación de Carrera en la Universidad de Guayaquil, se requiere fomentar nuevas estructuras y políticas de trabajo que mejores el funcionamiento de la misma, pues la educación requiere de cambios que fomenten el aprendizaje y las formas de enseñar, es por ello que se propone un plan de acciones que mejoren el trabajo del tutor y su superación personal desde la Dirección Técnica de la Nivelación de Carrera.

\section{Algunas consideraciones para mejorar el trabajo de los tutores en el Proyecto de Nivelación}

El objetivo final de la educación es la formación multilateral de las nuevas generaciones desde una concepción humanista. En el logro de este propósito es esencial la labor de los tutores y los académicos, garantizando un proceso de enseñanza aprendizaje desarrollador, que centre su atención en la formación y motivación de los estudiantes por las carreras que optan, por tanto, enfatiza en el aprendizaje de éste como elemento rector de la relación que se produce entre el profesor, tutor y sus alumnos.

Para el logro del propósito del Proyecto de Nivelación y del accionar de los tutores es importante que en su incidencia con los estudiantes logren potenciar un proceso de autorregulación de la personalidad de los mismos, implicándolos realmente en el proceso de aprendizaje y en su formación general.

Para favorecer esta adecuada formación inicial, continua y permanente del estudiante universitario es esencial potenciar los procesos reflexivos de los tutores, este debe constituir en un profesional reflexivo de su práctica, de su actuación profesional y personal, garantizando su autoeducación constante, o sea, debe adquirir la responsabilidad de orientarse a sí mismo y manejar su propia formación y evolución pedagógica.

La función pedagógica de los tutores se relaciona con las tareas de planificación, orientación, coordinación y control que realizan los grupos o paralelos que tiene a su cargo, para mejorar el desarrollo de las actividades docentes, incidir y realizar de forma coordinada las extradocentes y extraescolares, a través de los componentes académico e investigativo. Dentro de la dirección pedagógica el tutor debe atender, las necesidades, intereses de los estudiantes, así como brindarle una adecuada orientación en el camino que transitan en su formación para el ingreso a la educación superior.

El tutor cumple además una función educativa, través de la formación y desarrollo de sentimientos, actitudes, valores, normas de comportamientos y de relación con los demás en estudiantes y académicos, vinculados a las exigencias actuales recogidas en el Proyecto de Nivelación.

Se significa la orientación dentro de este proceso, concibiéndola como una orientación educativa, relacionada con la necesidad de que en este colectivo se promuevan los recursos personales y grupales para favorecer el autoconocimiento, autoeducación y auto perfeccionamiento constante de tutores y estudiantes. 


\section{Tareas y funciones de los tutores:}

1. Dominar sus tareas y funciones dentro de los grupos o paralelos donde incide.

2. Iniciar con la aplicación de un diagnóstico para determinar las características,necesidades e intereses de sus estudiantes (técnicas que considere).

3. Establecer lineamientos generales para la atención y seguimiento al diagnóstico de los estudiantes o grupos de la carrera donde incide.

4. Analizar los resultados del diagnóstico de cada grupo de la carrera, estableciendo pautas y objetivos generales para la elaboración de las estrategias educativas trabajo, considerado el contexto y las potencialidades.

5. Elaborar la estrategia educativa o plan de actividades para los grupos, a partir de las problemáticas y propuestas de solución, y en relación con los objetivos de la nivelación y de las facultades, incluyendo actividades que atiendan lo instructivo, educativo y orientador.

6. Coordinar, a través de las reuniones de trabajo de los tutores, la adecuada superación y preparación para el trabajo pedagógico, con énfasis en el trabajo colaborativo, en correspondencia con los objetivos de la nivelación, el diagnóstico y las estrategias que puedan desarrollarse, constituyéndose en un verdadero grupo de desarrollo.

7. Evaluar de forma sistemática la marcha del proceso educativo - pedagógico en los grupos, concibiendo reajustes en la estrategia educativa o plan de trabajo mensual, así como en el proceso de orientación y dirección que persiguen las reuniones de trabajo con los tutores según las condiciones lo requieran.

8. Prestar especial atención a los procesos grupales favoreciendo la unidad de acción del colectivo pedagógico, los tutores y la del propio grupo de estudiantes.

\section{Propuestas de temas para talleres de capacitación para los tutores del Proyecto de Nivelación que serán impartidos cada mes durante el período}

1. La orientación vocacional. Importancia de una adecuada orientación.

2. Orientación hacia la labor pedagógica del tutor. Papel y funciones de los tutores para el desarrollo del Proyecto de Nivelación.

3. Los problemas actuales de la pedagogía.

4. Principios para la dirección del proceso pedagógico de los tutores.

5. El diagnóstico integral como instrumento de la labor educativa.

6. La ética, los valores y el profesionalismo en la formación de los estudiantes universitarios. 
7. Las estrategias educativas como instrumento para dirigir la formación de la personalidad de los estudiantes.

8. La investigación científica y educacional. Importancia.

9. Las Etapas de la Investigación educacional.

\section{CONCLUSIONES}

1. El período de nivelación de carrera constituye un proceso de cambios para el estudiante, que le permite adentrase al mundo universitario con una concepción diferente de la educación.

2. El Tutor Pedagógico constituye una figura importante dentro del proceso de enseñanza aprendizaje en el período nivelatorio, ya que acompaña al estudiante durante el proceso de adaptación y asimilación de la vida universitaria, así como lo adentra en mundo investigativo, brindándoles las herramientas para la investigación.

3. La propuesta que se presenta constituye una herramienta inicial para ir perfeccionando el trabajo del Departamento de Nivelación de Carrera en general y del tutor en particular, en aras de mejor el proceso de enseñanza aprendizaje en la Universidad de Guayaquil y su unidad de Nivelación. 


\section{REFERENCIAS BIBLIOGRÁFICAS}

Addine,F. y García ,G (1995). Formación pedagógica general en la formación de los profesores. Curso pre-evento 1, Pedagogía'95, La Habana.

Benavides, V. y Vaquero, P. (2004): Sistema de acompañamiento y mejoramiento continuo. Municipio del Distrito Metropolitano de Quito. Dirección de Educación. Quito, Ecuador.

Colectivo de autores.(2000). Centro de Investigaciones y Documentación Educativa (CIDE). La Habana, Cuba.

Colectivo de autores. (2014). Instructivo de gestión Académica y Administrativa del curso de nivelación del SNNA.

Ferry, G. (1997). Pedagogía de la formación. Ediciones Novedades Educativas. Universidad de Buenos Aires. Buenos Aires.

Pérez , G. (2002). Metodología de la investigación educacional.

Editorial Pueble y Educación, La Habana, Cuba.

Santos, J. (2012). Instructivo para el curso de Nivelación de carrera en la UG. 